\title{
Continuity of Bounded Linear Operators on Normed Linear Spaces ${ }^{1]}$
}

\author{
Kazuhisa Nakasho \\ Yamaguchi University \\ Yamaguchi, Japan
}

\author{
Yuichi Futa \\ Tokyo University of Technology \\ Tokyo, Japan
}

\author{
Yasunari Shidama \\ Shinshu University \\ Nagano, Japan
}

\begin{abstract}
Summary. In this article, using the Mizar system [1, 2], we discuss the continuity of bounded linear operators on normed linear spaces. In the first section, it is discussed that bounded linear operators on normed linear spaces are uniformly continuous and Lipschitz continuous. Especially, a bounded linear operator on the dense subset of a complete normed linear space has a unique natural extension over the whole space. In the next section, several basic currying properties are formalized.

In the last section, we formalized that continuity of bilinear operator is equivalent to both Lipschitz continuity and local continuity. We referred to [4, 13, and [3] in this formalization.
\end{abstract}

MSC: 46-00 47A07 47A30 68T99 03B35

Keywords: Lipschitz continuity; uniform continuity; bounded linear operators; bilinear operators

MML identifier: LOPBAN_8, version: 8.1 .08 5.53.1335

\section{Uniform Continuity and Lipschitz Continuity of Bounded LiNEAR OPERATORS}

From now on $S, T, W, Y$ denote real normed spaces, $f$ denotes partial function from $S$ to $T, Z$ denotes a subset of $S$, and $i, n$ denote natural numbers.

\footnotetext{
${ }^{1}$ This study was supported in part by JSPS KAKENHI Grant Number JP17K00182. 
Now we state the propositions:

(1) Let us consider real normed spaces $E, F$, a subset $E_{1}$ of $E$, and a partial function $f$ from $E$ to $F$. Suppose $E_{1}$ is dense and $F$ is complete and $\operatorname{dom} f=E_{1}$ and $f$ is uniformly continuous on $E_{1}$. Then

(i) there exists a function $g$ from $E$ into $F$ such that $g\left\lceil E_{1}=f\right.$ and $g$ is uniformly continuous on the carrier of $E$ and for every point $x$ of $E$, there exists a sequence $s_{0}$ of $E$ such that $\operatorname{rng} s_{0} \subseteq E_{1}$ and $s_{0}$ is convergent and $\lim s_{0}=x$ and $f_{*} s_{0}$ is convergent and $g(x)=$ $\lim \left(f_{*} s_{0}\right)$ and for every point $x$ of $E$ and for every sequence $s_{0}$ of $E$ such that rng $s_{0} \subseteq E_{1}$ and $s_{0}$ is convergent and $\lim s_{0}=x$ holds $f_{*} s_{0}$ is convergent and $g(x)=\lim \left(f_{*} s_{0}\right)$, and

(ii) for every functions $g_{1}, g_{2}$ from $E$ into $F$ such that $g_{1}\left\lceil E_{1}=f\right.$ and $g_{1}$ is continuous on the carrier of $E$ and $g_{2}\left\lceil E_{1}=f\right.$ and $g_{2}$ is continuous on the carrier of $E$ holds $g_{1}=g_{2}$.

Proof: For every point $x$ of $E$ and for every sequence $s_{0}$ of $E$ such that $\operatorname{rng} s_{0} \subseteq E_{1}$ and $s_{0}$ is convergent for every real number $s$ such that $0<s$ there exists a natural number $n$ such that for every natural number $m$ such that $n \leqslant m$ holds $\left\|\left(f_{*} s_{0}\right)(m)-\left(f_{*} s_{0}\right)(n)\right\|<s$. For every point $x$ of $E$ and for every sequence $s_{0}$ of $E$ such that $\mathrm{rng} s_{0} \subseteq E_{1}$ and $s_{0}$ is convergent holds $f_{*} s_{0}$ is convergent by $[12,(5)]$. For every point $x$ of $E$ and for every sequences $s_{1}, s_{2}$ of $E$ such that $\operatorname{rng} s_{1} \subseteq E_{1}$ and $s_{1}$ is convergent and $\lim s_{1}=x$ and $\operatorname{rng} s_{2} \subseteq E_{1}$ and $s_{2}$ is convergent and $\lim s_{2}=x$ holds $\lim \left(f_{*} s_{1}\right)=\lim \left(f_{*} s_{2}\right)$ by [7, (14)].

Define $\mathcal{P}$ [object, object $] \equiv$ there exists a sequence $s_{0}$ of $E$ such that $\operatorname{rng} s_{0} \subseteq E_{1}$ and $s_{0}$ is convergent and $\lim s_{0}=\$_{1}$ and $f_{*} s_{0}$ is convergent and $\$_{2}=\lim \left(f_{*} s_{0}\right)$. For every element $x$ of $E$, there exists an element $y$ of $F$ such that $\mathcal{P}[x, y]$. Consider $g$ being a function from $E$ into $F$ such that for every element $x$ of $E, \mathcal{P}[x, g(x)]$. For every object $x$ such that $x \in \operatorname{dom} f$ holds $f(x)=g(x)$. For every point $x$ of $E$ and for every sequence $s_{0}$ of $E$ such that $\operatorname{rng} s_{0} \subseteq E_{1}$ and $s_{0}$ is convergent and $\lim s_{0}=x$ holds $f_{*} s_{0}$ is convergent and $g(x)=\lim \left(f_{*} s_{0}\right)$. For every real number $r$ such that $0<r$ there exists a real number $s$ such that $0<s$ and for every points $x_{1}, x_{2}$ of $E$ such that $x_{1}, x_{2} \in$ the carrier of $E$ and $\left\|x_{1}-x_{2}\right\|<s$ holds $\left\|g_{/ x_{1}}-g_{/ x_{2}}\right\|<r$. For every element $x$ of $E, g_{1}(x)=g_{2}(x)$ by [5. (14)], [9, (18)].

(2) Let us consider real normed spaces $E, F, G$, a point $f$ of the real norm space of bounded linear operators from $E$ into $F$, and a point $g$ of the real norm space of bounded linear operators from $F$ into $G$. Then there exists a point $h$ of the real norm space of bounded linear operators from $E$ into 
$G$ such that

(i) $h=g \cdot f$, and

(ii) $\|h\| \leqslant\|g\| \cdot\|f\|$.

Proof: Reconsider $L_{1}=f$ as a Lipschitzian linear operator from $E$ into $F$. Reconsider $L_{2}=g$ as a Lipschitzian linear operator from $F$ into $G$. Set $L_{3}=L_{2} \cdot L_{1}$. For every real number $t$ such that $t \in \operatorname{PreNorms}\left(L_{3}\right)$ holds $t \leqslant\|g\| \cdot\|f\|$ by [11, (16)].

(3) Let us consider real normed spaces $E, F$. Then every Lipschitzian linear operator from $E$ into $F$ is Lipschitzian on the carrier of $E$ and uniformly continuous on the carrier of $E$.

Proof: Consider $K$ being a real number such that $0 \leqslant K$ and for every vector $x$ of $E,\|L(x)\| \leqslant K \cdot\|x\|$. Set $r=K+1$. Set $E_{0}=$ the carrier of $E$. For every points $x_{1}, x_{2}$ of $E$ such that $x_{1}, x_{2} \in E_{0}$ holds $\left\|L_{/ x_{1}}-L / x_{2}\right\| \leqslant$ $r \cdot\left\|x_{1}-x_{2}\right\|$.

(4) Let us consider real normed spaces $E, F$, a subreal normal space $E_{1}$ of $E$, and a point $f$ of the real norm space of bounded linear operators from $E_{1}$ into $F$. Suppose $F$ is complete and there exists a subset $E_{0}$ of $E$ such that $E_{0}=$ the carrier of $E_{1}$ and $E_{0}$ is dense. Then

(i) there exists a point $g$ of the real norm space of bounded linear operators from $E$ into $F$ such that dom $g=$ the carrier of $E$ and $g \uparrow\left(\right.$ the carrier of $\left.E_{1}\right)=f$ and $\|g\|=\|f\|$ and there exists a partial function $L_{1}$ from $E$ to $F$ such that $L_{1}=f$ and for every point $x$ of $E$, there exists a sequence $s_{0}$ of $E$ such that rng $s_{0} \subseteq$ the carrier of $E_{1}$ and $s_{0}$ is convergent and $\lim s_{0}=x$ and $L_{1 *} s_{0}$ is convergent and $g(x)=\lim \left(L_{1 *} s_{0}\right)$ and for every point $x$ of $E$ and for every sequence $s_{0}$ of $E$ such that rng $s_{0} \subseteq$ the carrier of $E_{1}$ and $s_{0}$ is convergent and $\lim s_{0}=x$ holds $L_{1 *} s_{0}$ is convergent and $g(x)=\lim \left(L_{1 *} s_{0}\right)$, and

(ii) for every points $g_{1}, g_{2}$ of the real norm space of bounded linear operators from $E$ into $F$ such that $g_{1} \uparrow\left(\right.$ the carrier of $\left.E_{1}\right)=f$ and $g_{2} \uparrow\left(\right.$ the carrier of $\left.E_{1}\right)=f$ holds $g_{1}=g_{2}$.

Proof: Consider $E_{0}$ being a subset of $E$ such that $E_{0}=$ the carrier of $E_{1}$ and $E_{0}$ is dense. Reconsider $L=f$ as a Lipschitzian linear operator from $E_{1}$ into $F$. Reconsider $L_{1}=L$ as a partial function from $E$ to $F$. Consider $K$ being a real number such that $0 \leqslant K$ and for every vector $x$ of $E_{1},\|L(x)\| \leqslant K \cdot\|x\|$. Set $r=K+1$. For every points $x_{1}, x_{2}$ of $E$ such that $x_{1}, x_{2} \in E_{0}$ holds $\left\|L_{1 / x_{1}}-L_{1 / x_{2}}\right\| \leqslant r \cdot\left\|x_{1}-x_{2}\right\|$.

There exists a function $P_{3}$ from $E$ into $F$ such that $P_{3}\left\lceil E_{0}=L_{1}\right.$ and $P_{3}$ is uniformly continuous on the carrier of $E$ and for every point 
$x$ of $E$, there exists a sequence $s_{0}$ of $E$ such that $\operatorname{rng} s_{0} \subseteq E_{0}$ and $s_{0}$ is convergent and $\lim s_{0}=x$ and $L_{1 *} s_{0}$ is convergent and $P_{3}(x)=\lim \left(L_{1 *} s_{0}\right)$ and for every point $x$ of $E$ and for every sequence $s_{0}$ of $E$ such that $\operatorname{rng} s_{0} \subseteq E_{0}$ and $s_{0}$ is convergent and $\lim s_{0}=x$ holds $L_{1 *} s_{0}$ is convergent and $P_{3}(x)=\lim \left(L_{1 *} s_{0}\right)$ and for every functions $P_{1}, P_{2}$ from $E$ into $F$ such that $P_{1}\left\lceil E_{0}=L_{1}\right.$ and $P_{1}$ is continuous on the carrier of $E$ and $P_{2}\left\lceil E_{0}=L_{1}\right.$ and $P_{2}$ is continuous on the carrier of $E$ holds $P_{1}=P_{2}$.

Consider $P_{3}$ being a function from $E$ into $F$ such that $P_{3}\left\lceil E_{0}=L_{1}\right.$ and $P_{3}$ is uniformly continuous on the carrier of $E$ and for every point $x$ of $E$, there exists a sequence $s_{0}$ of $E$ such that $\operatorname{rng} s_{0} \subseteq E_{0}$ and $s_{0}$ is convergent and $\lim s_{0}=x$ and $L_{1 *} s_{0}$ is convergent and $P_{3}(x)=\lim \left(L_{1 *} s_{0}\right)$ and for every point $x$ of $E$ and for every sequence $s_{0}$ of $E$ such that $\operatorname{rng} s_{0} \subseteq E_{0}$ and $s_{0}$ is convergent and $\lim s_{0}=x$ holds $L_{1 *} s_{0}$ is convergent and $P_{3}(x)=\lim \left(L_{1 *} s_{0}\right)$ and for every point $x$ of $E$, there exists a sequence $s_{0}$ of $E$ such that $\operatorname{rng} s_{0} \subseteq E_{0}$ and $s_{0}$ is convergent and $\lim s_{0}=x$ and $L_{1 *} s_{0}$ is convergent and $P_{3}(x)=\lim \left(L_{1 *} s_{0}\right)$ and for every point $x$ of $E$ and for every sequence $s_{0}$ of $E$ such that $\operatorname{rng} s_{0} \subseteq E_{0}$ and $s_{0}$ is convergent and $\lim s_{0}=x$ holds $L_{1 *} s_{0}$ is convergent and $P_{3}(x)=\lim \left(L_{1 *} s_{0}\right)$. For every points $x, y$ of $E, P_{3}(x+y)=P_{3}(x)+P_{3}(y)$. For every point $x$ of $E$ and for every real number $a, P_{3}(a \cdot x)=a \cdot P_{3}(x)$.

Reconsider $g=P_{3}$ as a point of the real norm space of bounded linear operators from $E$ into $F$. For every real number $t$ such that $t \in$ PreNorms $(L)$ holds $t \leqslant\|g\|$. For every real number $t$ such that $t \in$ $\operatorname{PreNorms}\left(P_{3}\right)$ holds $t \leqslant\|f\|$. For every points $g_{1}, g_{2}$ of the real norm space of bounded linear operators from $E$ into $F$ such that $g_{1} \uparrow$ (the carrier of $\left.E_{1}\right)=f$ and $g_{2} \uparrow\left(\right.$ the carrier of $\left.E_{1}\right)=f$ holds $g_{1}=g_{2}$ by (3), [8, (7)], (1).

\section{Basic Properties of Currying}

Now we state the propositions:

(5) Let us consider non empty sets $E, F, G$, a function $f$ from $E \times F$ into $G$, and an object $x$. If $x \in E$, then (curry $f)(x)$ is a function from $F$ into $G$.

(6) Let us consider non empty sets $E, F, G$, a function $f$ from $E \times F$ into $G$, and an object $y$. If $y \in F$, then $\left(\operatorname{curry}^{\prime} f\right)(y)$ is a function from $E$ into G.

Let us consider non empty sets $E, F, G$, a function $f$ from $E \times F$ into $G$, and objects $x, y$. Now we state the propositions: 
(7) If $x \in E$ and $y \in F$, then $(\operatorname{curry} f)(x)(y)=f(x, y)$.

(8) If $x \in E$ and $y \in F$, then $\left(\right.$ curry $\left.^{\prime} f\right)(y)(x)=f(x, y)$.

Let $E, F, G$ be real linear spaces and $f$ be a function from (the carrier of $E) \times($ the carrier of $F$ ) into the carrier of $G$. We say that $f$ is bilinear if and only if

(Def. 1) for every point $v$ of $E$ such that $v \in \operatorname{dom}(\operatorname{curry} f)$ holds (curry $f)(v)$ is an additive, homogeneous function from $F$ into $G$ and for every point $v$ of $F$ such that $v \in \operatorname{dom}\left(\operatorname{curry}^{\prime} f\right)$ holds $\left(\right.$ curry $\left.^{\prime} f\right)(v)$ is an additive, homogeneous function from $E$ into $G$.

\section{Equivalence of Some Definitions of Continuity of Bilinear OPERATORS}

Now we state the proposition:

(9) Let us consider real linear spaces $E, F, G$. Then (the carrier of $E$ ) $\times$ (the carrier of $F) \longmapsto 0_{G}$ is bilinear.

Proof: Set $f=($ the carrier of $E) \times($ the carrier of $F) \longmapsto 0_{G}$. For every point $x$ of $E$, (curry $f)(x)$ is an additive, homogeneous function from $F$ into $G$. For every point $x$ of $F$ such that $x \in \operatorname{dom}\left(\operatorname{curry}^{\prime} f\right)$ holds $\left(\right.$ curry $\left.^{\prime} f\right)(x)$ is an additive, homogeneous function from $E$ into $G$.

Let $E, F, G$ be real linear spaces. Observe that there exists a function from (the carrier of $E) \times($ the carrier of $F$ ) into the carrier of $G$ which is bilinear.

Now we state the proposition:

(10) Let us consider real linear spaces $E, F, G$, and a function $L$ from (the carrier of $E) \times($ the carrier of $F$ ) into the carrier of $G$. Then $L$ is bilinear if and only if for every points $x_{1}, x_{2}$ of $E$ and for every point $y$ of $F, L\left(x_{1}+x_{2}, y\right)=L\left(x_{1}, y\right)+L\left(x_{2}, y\right)$ and for every point $x$ of $E$ and for every point $y$ of $F$ and for every real number $a, L(a \cdot x, y)=a \cdot L(x, y)$ and for every point $x$ of $E$ and for every points $y_{1}, y_{2}$ of $F, L\left(x, y_{1}+y_{2}\right)=$ $L\left(x, y_{1}\right)+L\left(x, y_{2}\right)$ and for every point $x$ of $E$ and for every point $y$ of $F$ and for every real number $a, L(x, a \cdot y)=a \cdot L(x, y)$. The theorem is a consequence of (8) and (7).

Let $E, F, G$ be real linear spaces and $f$ be a function from $E \times F$ into $G$. We say that $f$ is bilinear if and only if

(Def. 2) there exists a function $g$ from (the carrier of $E) \times($ the carrier of $F$ ) into the carrier of $G$ such that $f=g$ and $g$ is bilinear.

One can verify that there exists a function from $E \times F$ into $G$ which is bilinear. 
Let $f$ be a function from $E \times F$ into $G, x$ be a point of $E$, and $y$ be a point of $F$. Note that the functor $f(x, y)$ yields a point of $G$. Now we state the proposition:

(11) Let us consider real linear spaces $E, F, G$, and a function $L$ from $E \times$ $F$ into $G$. Then $L$ is bilinear if and only if for every points $x_{1}, x_{2}$ of $E$ and for every point $y$ of $F, L\left(x_{1}+x_{2}, y\right)=L\left(x_{1}, y\right)+L\left(x_{2}, y\right)$ and for every point $x$ of $E$ and for every point $y$ of $F$ and for every real number $a$, $L(a \cdot x, y)=a \cdot L(x, y)$ and for every point $x$ of $E$ and for every points $y_{1}$, $y_{2}$ of $F, L\left(x, y_{1}+y_{2}\right)=L\left(x, y_{1}\right)+L\left(x, y_{2}\right)$ and for every point $x$ of $E$ and for every point $y$ of $F$ and for every real number $a, L(x, a \cdot y)=a \cdot L(x, y)$.

Let $E, F, G$ be real linear spaces.

A bilinear operator from $E \times F$ into $G$ is a bilinear function from $E \times F$ into $G$. Let $E, F, G$ be real normed spaces and $f$ be a function from $E \times F$ into $G$. We say that $f$ is bilinear if and only if

(Def. 3) there exists a function $g$ from (the carrier of $E) \times($ the carrier of $F$ ) into the carrier of $G$ such that $f=g$ and $g$ is bilinear.

Let us note that there exists a function from $E \times F$ into $G$ which is bilinear.

A bilinear operator from $E \times F$ into $G$ is a bilinear function from $E \times F$ into $G$. From now on $E, F, G$ denote real normed spaces, $L$ denotes a bilinear operator from $E \times F$ into $G, x$ denotes an element of $E$, and $y$ denotes an element of $F$.

Let $E, F, G$ be real normed spaces, $f$ be a function from $E \times F$ into $G, x$ be a point of $E$, and $y$ be a point of $F$. Note that the functor $f(x, y)$ yields a point of $G$. Now we state the propositions:

(12) Let us consider real normed spaces $E, F, G$, and a function $L$ from $E \times$ $F$ into $G$. Then $L$ is bilinear if and only if for every points $x_{1}, x_{2}$ of $E$ and for every point $y$ of $F, L\left(x_{1}+x_{2}, y\right)=L\left(x_{1}, y\right)+L\left(x_{2}, y\right)$ and for every point $x$ of $E$ and for every point $y$ of $F$ and for every real number $a$, $L(a \cdot x, y)=a \cdot L(x, y)$ and for every point $x$ of $E$ and for every points $y_{1}$, $y_{2}$ of $F, L\left(x, y_{1}+y_{2}\right)=L\left(x, y_{1}\right)+L\left(x, y_{2}\right)$ and for every point $x$ of $E$ and for every point $y$ of $F$ and for every real number $a, L(x, a \cdot y)=a \cdot L(x, y)$.

(13) Let us consider real normed spaces $E, F, G$, and a bilinear operator $f$ from $E \times F$ into $G$. Then

(i) $f$ is continuous on the carrier of $E \times F$ iff $f$ is continuous in $0_{E \times F}$, and

(ii) $f$ is continuous on the carrier of $E \times F$ iff there exists a real number $K$ such that $0 \leqslant K$ and for every point $x$ of $E$ and for every point $y$ of $F,\|f(x, y)\| \leqslant K \cdot\|x\| \cdot\|y\|$. 
Proof: If $f$ is continuous in $0_{E \times F}$, then there exists a real number $K$ such that $0 \leqslant K$ and for every point $x$ of $E$ and for every point $y$ of $F$, $\|f(x, y)\| \leqslant K \cdot\|x\| \cdot\|y\|$ by [9, (7)], [6, (22)], [10, (18)]. If there exists a real number $K$ such that $0 \leqslant K$ and for every point $x$ of $E$ and for every point $y$ of $F,\|f(x, y)\| \leqslant K \cdot\|x\| \cdot\|y\|$, then $f$ is continuous on the carrier of $E \times F$.

\section{REFERENCES}

[1] Grzegorz Bancerek, Czesław Byliński, Adam Grabowski, Artur Korniłowicz, Roman Matuszewski, Adam Naumowicz, Karol Pąk, and Josef Urban. Mizar: State-of-the-art and beyond In Manfred Kerber, Jacques Carette, Cezary Kaliszyk, Florian Rabe, and Volker Sorge, editors, Intelligent Computer Mathematics, volume 9150 of Lecture Notes in Computer Science, pages 261-279. Springer International Publishing, 2015. ISBN 978-3319-20614-1. doi 10.1007/978-3-319-20615-8_17.

[2] Grzegorz Bancerek, Czesław Byliński, Adam Grabowski, Artur Korniłowicz, Roman Matuszewski, Adam Naumowicz, and Karol Pąk. The role of the Mizar Mathematical Library for interactive proof development in Mizar. Journal of Automated Reasoning, 61(1):9-32, 2018. do1 $10.1007 / \mathrm{s} 10817-017-9440-6$

[3] N. J. Dunford and T. Schwartz. Linear operators I. Interscience Publ., 1958.

[4] Miyadera Isao. Functional Analysis. Riko-Gaku-Sya, 1972.

[5] Kazuhisa Nakasho, Yuichi Futa, and Yasunari Shidama. Topological properties of real normed space. Formalized Mathematics, 22(3):209-223, 2014. doi 10.2478/forma-20140024 .

[6] Kazuhisa Nakasho, Yuichi Futa, and Yasunari Shidama. Implicit function theorem. Part I. Formalized Mathematics, 25(4):269-281, 2017. doi:10.1515/forma-2017-0026.

[7] Keiko Narita, Noboru Endou, and Yasunari Shidama. Riemann integral of functions from $\mathbb{R}$ into real Banach space. Formalized Mathematics, 21(2):145-152, 2013. doi $10.2478 /$ forma-2013-0016.

[8] Takaya Nishiyama, Artur Korniłowicz, and Yasunari Shidama. The uniform continuity of functions on normed linear spaces. Formalized Mathematics, 12(3):277-279, 2004.

[9] Takaya Nishivama, Keiji Ohkubo, and Yasunari Shidama. The continuous functions on normed linear spaces Formalized Mathematics, 12(3):269-275, 2004.

[10] Hiroyuki Okazaki, Noboru Endou, and Yasunari Shidama. Cartesian products of family of real linear spaces. Formalized Mathematics, 19(1):51-59, 2011. doi:10.2478/v10037011-0009-2.

[11] Yasunari Shidama. Banach space of bounded linear operators Formalized Mathematics, 12(1):39-48, 2004.

[12] Yasunari Shidama. The series on Banach algebra. Formalized Mathematics, 12(2):131138, 2004.

[13] Kosaku Yoshida. Functional Analysis. Springer, 1980.

Accepted September 29, 2018 